\title{
Schöpf-Schulz-Passarge syndrome
}

\author{
INSERM
}

\section{Source}

INSERM. (1999). Orphanet: an online rare disease and orphan drug data base. SchöpfSchulz-Passarge syndrome. ORPHA:50944

Schöpf-Schulz-Passarge syndrome (SSPS) is a rare autosomal recessive ectodermal dysplasia characterized by multiple eyelid apocrine hidrocystomas, palmoplantar keratoderma, hypotrichosis, hypodontia and nail dystrophy. 